\title{
Author Correction: Chloroplast FtsZ assembles into a contractible ring via tubulin-like heteropolymerization
}

\author{
Yamato Yoshida, Yuko Mogi, Allan D. TerBush and Katherine W. Osteryoung
}

Correction to: Nature Plants https://doi.org/10.1038/nplants.2016.95, published online 20 June 2016.

In the version of this Article originally published, the authors incorrectly referred to the fluorescent protein Venus being used in their study; the actual one used was enhanced yellow fluorescence protein (eYFP).

Published online: 12 December 2018

https://doi.org/10.1038/s41477-018-0341-4 